\title{
Naturally Chiral Surfaces
}

\author{
Joshua D. Horvath and Andrew J. Gellman* \\ Department of Chemical Engineering \\ Carnegie Mellon University \\ Pittsburgh, PA 15213, USA
}

\begin{abstract}
Chiral surfaces are of growing importance as a result of their potential for use in enantioselective chemical processes. By far the most widely used and commonly studied chiral surfaces are those prepared by templating of achiral surface with chiral organic ligands. It is possible, however, to prepare naturally chiral surfaces by a number of means. This paper describes the various types of chiral surfaces. In addition data are presented to suggest that naturally chiral surface of metals can be prepared by a process of imprinting in which chiral adsorbates induce reconstructions that create chiral kinks on metal surfaces.
\end{abstract}

* corresponding author, (412-268-3848), gellman@cmu.edu 


\section{Introduction}

Any two objects which are nonsuperimposable mirror images of one another are chiral. Chirality manifests itself in many different forms and at all length scales throughout nature. Ones' left and right hands are the most familiar examples, however, there are many more. In molecules chirality appears most frequently in the form of carbon atoms that are tetrahedrally coordinated to four different substituents. Two such molecules with different arrangements of their four substituents are nonsuperimposable images of one another. Although these two molecules have many identical properties this seemingly innocuous structural difference can have profound effects when they interact with other chiral molecules or chiral environments. This is critically important in living organisms because almost all bioactive molecules such as proteins and enzymes are chiral and exist in only one enantiomeric form. Thus the left and right handed enantiomers of a chiral compound will have different physiological effects in the human body simply because they interact in different ways with the enzymes and proteins, all of which are of one handedness.

Many pharmaceuticals have structures containing tetrahedrally coordinated carbon atoms that are chiral. As a consequence they exist in left and right handed forms and it is only one of the two enantiomers that is therapeutically active when ingested. More often than not, the other enantiomer is toxic. Thus there are enormous real and regulatory pressures for pharmaceutical manufacturers to produce single enantiomer forms of most pharmaceuticals.

Under most conditions a chemical reaction that produces a chiral product will produce both enantiomers in equal quantities, forming what is known as a racemic mixture. To produce pharmaceuticals that are enantiomerically pure requires chemical processing steps that are enantioselective. These could be synthesis steps that produce only one of the two enantiomers or separation steps that are enantioselective. In either case these enantioselective chemical processes must occur in chiral environments of a single handedness: enantiomerically pure reagents, chiral solvents, enzymes, chiral adsorbents, or chiral catalysts. Furthermore, the chirality of these environments must occur on length scales that are of molecular dimensions in order for the interaction of the 
reagents to be enantiospecific. Many chemical processing steps involve surfaces as catalytic sites or for adsorption. If these surfaces can be prepared such that they have chiral structures, then catalysis or adsorption on them can be enantiospecific and can lead to enantioselectivity. This paper discusses the possible origins of chirality at the surfaces of solid materials and means for preparing homochiral surfaces. Some such surfaces occur by cleaving of materials that have bulk chiral structures, however, it is also possible for materials such as common catalytic metals which have achiral bulk structures to expose surfaces that are naturally chiral.

\section{Bulk Chiral Crystals}

Naturally occurring mineral crystals that do not have a center of symmetry in their bulk structures can expose chiral surfaces. Quartz is the most commonly found chiral material in nature, and its chirality arises from the helical arrangement of $\mathrm{SiO}_{4}$ tetrahedra in the bulk structure ${ }^{1}$. Since the bulk structure of quartz is chiral its surfaces must be chiral and must exhibit enantiospecific interaction with the two enantiomers of a chiral compound. Bonner et al. examined the adsorption of radioactive D- and L-alanine hydrochloride on D- and L-quartz crystals ${ }^{2}$. The radioactivity of powdered samples of the chiral quartz crystals after exposure to the alanine was used to quantify adsorption of the amino acids onto the quartz surfaces. D-alanine adsorbed preferentially onto the Dquartz while L-alanine adsorbed preferentially onto the L-quartz, with an enantiomeric excess adsorption of between 1.0 and 1.8 percent in each case. Enantioselective adsorption has also been observed on other mineral surfaces with even higher selectivity. Hazen et al. studied the adsorption of chiral molecules on calcite, an achiral mineral that can expose chiral crystal faces ${ }^{3}$. Amino acids are known to have high heats of adsorption on calcite, making them ideal probes for enantioselective adsorption. Exposure of D- and L-aspartic acid to the chiral $(21 \overline{3} 1)$ and $(3 \overline{1} \overline{2} 1)$ faces of calcite resulted in a chiral excess adsorption of approximately $10 \%$. Adsorption of D-aspartic acid was favored on the $(21 \overline{3} 1)$ surface, while L-aspartic acid adsorption was favored on the $(3 \overline{1} \overline{2} 1)$ surface. A control experiment was performed using the achiral (10 $\overline{1} 1)$ surface which did not selectively adsorb either D- or L-aspartic acid. In addition to exhibiting enantioselective adsorption, chiral quartz powders have been used to nucleate an autocatalytic synthesis of 
a pyramidal alkanols to produce an enantiomeric excess of $e e>90 \%{ }^{4}$. Thus enantioselective surface chemistry has been demonstrated on the surfaces of a number of materials with naturally chiral bulk structures.

\section{Chirally templated surfaces}

The most common method for preparation of chiral surfaces is by adsorption of a chiral organic ligand onto and otherwise achiral surface. The resulting surface is chiral if the adsorbed ligand retains its chirality. Chirality can be imparted to templated surfaces in two ways. On the one hand the intrinsic chirality of the ligand creates a chiral environment on the surface in the immediate vicinity of its adsorption site. Alternatively, the adsorbed ligands can assemble into ordered arrays with a chiral unit cell imparting long range chirality to the surface. Adsorption of $\mathrm{R}, \mathrm{R}$-tartaric acid on $\mathrm{Cu}(110)$ results in an ordered overlayer structure under certain conditions, imparting chirality to the achiral $\mathrm{Cu}(110)$ surface $^{5}$. Similar results have been observed for the creation of chiral surfaces by adsorption of R,R-tartaric acid on $\mathrm{Ni}(110)^{6}$ and $\mathrm{Ni}(111)^{7}$. The importance of tartaric acid is that it can be used to modify the surfaces of powdered Ni catalysts which are then used for the enantioselective hydrogenation of $\beta$-ketoesters. Perhaps the most carefully studied enantioselective heterogeneous catalyst is based on Pt modified by templating with cinchonidine. These catalysts can be used for the hydrogenation of $\alpha$-ketoesters and can achieve enantioselectivities of $e e>95 \%$ under certain conditions. Cinchonidine has been shown to form an ordered overlayer structure on $\mathrm{Cu}(111)^{8}$, although no ordered structure was observed on $\operatorname{Pt}(111)^{9}$. Even larger molecules have been used to create templated chiral surfaces. Heptahelicene $\left(\mathrm{C}_{30} \mathrm{H}_{18}\right)$ adsorbed on $\mathrm{Cu}(332)$ creates a chiral surface with single-phase orientational order $^{10}$.

All the amino acids other than glycine are chiral and a number of them have been studied on achiral metal surfaces. Adsorbed alanine forms several different order phases on the $\mathrm{Cu}(110)$ surface at different coverages and temperatures. A two-dimensional homochiral phase can be observed under some conditions ${ }^{11}$. R- and S-phenylglycine also form chiral domains with long range order on the $\mathrm{Cu}(110)$ surface whose chirality can be observed using low energy electron diffraction ${ }^{12}$. The chiral amino acid S-proline forms 
an ordered overlayer structure on the $\mathrm{Cu}(110)$ surface, but does not exhibit the variety of phases observed for alanine ${ }^{13}$.

Most surface science studies of chirally templated surfaces have been motivated by the desire to understand the behavior of chirally templated heterogeneous catalysts and have focused on the surface chemistry and structure of the templating agent itself.

Although the surfaces described above are chiral, none have yet been used to demonstrate enantioselective surface chemistry. One example of an enantiospecific process that has been studied on a chirally templated single crystal metal surface is the enantioselective chemisorption of propylene oxide on a $\mathrm{Pd}(111)$ surface modified by adsorption of chiral 2-butanoxy groups ${ }^{14}$. The 2-butanoxy groups were formed by dissociative adsorption of R- and S-2-butanol. Subsequent exposure of the surface to R-and S-propylene oxide resulted in enantiospecific adsorption. Twice as much R-propylene oxide adsorbed on the $\operatorname{Pd}(111)$ surface modified with R-2-butanoxide as on the $\operatorname{Pd}(111)$ surface modified with S-2-butanoxide. All such chirally templated surfaces ought to exhibit enantioselectivity in the presence of chiral adsorbates and surface science experiment will enable us to understand the origins of enantioselectivity in depth, however, such investigations are in their infancy.

\section{Naturally chiral surfaces of achiral materials}

Metals have achiral bulk crystal structures, and so it is somewhat counter intuitive that they can expose surfaces that are naturally chiral. Nonetheless, high Miller index metal surfaces that expose kinked steps are in fact chiral. The chirality of this type of surface arises from the chiral structure of the kink. The $\mathrm{Cu}(643)$ and $\mathrm{Cu}(\overline{6} \overline{4} \overline{3})$ surfaces are illustrated in Figure 1 and are nonsuperimposable mirror images of one another and, therefore, are chiral. The kinks on these surfaces are formed by the intersection of (100), (110), and (111) microfacets at the surface which can be arranged with either a clockwise or a counterclockwise sense of rotation as one looks down on the surface. This sense of orientation renders the surfaces chiral and serves as the basis for a nomenclature that is used to designate these two surfaces as either $\mathrm{Cu}(643)^{\mathrm{S}}$ or $\mathrm{Cu}(643)^{\mathrm{R}} 15,16$.

The structures of the $\mathrm{Cu}(643)^{\mathrm{R}}$ and $\mathrm{Cu}(643)^{\mathrm{S}}$ surfaces depicted in Figure 1 are based on the ideal terminations of the face-centered cubic bulk lattice along the (643) plane and 
are not likely to represent the structures of real surfaces. The ideal structure has single atom kinks with (110) structure separated by (100) step edges which are two atoms in length. At temperature above absolute zero atoms can diffuse across a real surface producing non-ideal structures as a results of thermal roughening effects. Our current understanding of the real structure of these naturally chiral surface comes from simulations of thermal roughening effects on $\operatorname{Pt}(643)$ surfaces. These simulations indicate that thermal roughening leads to structures similar to that shown in the inset of Figure $2^{15,17,18}$. Atoms diffuse along step edges causing kinks to coalesce so that rather than having a periodic array of kinks one has them randomly distributed along the step edge. Furthermore the kinks are no longer just single atom kinks and can be separated by long step edges. The net effect is to reduce the number of kinks and to cause the appearance of non-ideal kinks at the intersections of long step edges. Nonetheless there is no significant generation of kinks that are of opposite handedness to those on the perfect, unrelaxed surface. Scanning tunneling microscopy has confirmed that real $\mathrm{Cu}(643)$ surfaces at room temperature have a structures that are in qualitative agreement with those predicted simulations of thermal roughening ${ }^{19}$. Thus although one does not expect real high Miller index surfaces of metals to have the ideal structure of the bulk termination plane they are chiral.

Sholl et al. have used atomistic simulations to probe the interactions of small chiral hydrocarbons with chiral Pt surfaces ${ }^{15}$. Adsorption of trans-1,2-dimethylcyclopropane, trans-1,2-dimethylcyclobutane, trans-1,2-dimethylcyclopentane, and trans-1,2dimethylcyclohexane was examined on the $\operatorname{Pt}(643), \operatorname{Pt}(531), \operatorname{Pt}(854)$, and $\operatorname{Pt}(874)$ surfaces. Enantiomeric differences in the adsorption energies of the two enantiomers of the same chiral compound on a given chiral Pt surface ranged from 0.0 to $0.7 \mathrm{kcal} / \mathrm{mole}$. In 9 of the 32 cases examined by Sholl et al., the enantiospecific adsorption energy differences were below the limits of experimental detection of $\sim 0.1 \mathrm{kcal} / \mathrm{mole}$. These theoretical results suggest that in some cases (but not all), enantiospecific adsorption energy differences can be large enough to measure experimentally.

Several chiral adsorbate have been adsorbed on the naturally chiral $\mathrm{Cu}(643)$ surfaces in order to study enantioselectivity. R-3-methylcyclohexanone (R-3-MCHO) was found to exhibit measurable enantiospecific differences in its desorption energy from the 
$\mathrm{Cu}(643)^{\mathrm{R}}$ and $\mathrm{Cu}(643)^{\mathrm{S}}$ surfaces $^{20-22}$. Figure 2 illustrates a temperature-programmed desorption (TPD) spectrum obtained by desorption of a saturated monolayer of R-3MCHO from the $\mathrm{Cu}(643)^{\mathrm{S}}$ surface. The TPD spectrum exhibits three distinct desorption peaks which can be associated with the desorption of R-3-MCHO from three distinct adsorption sites on the surface, as indicated in the inset to Figure 2. A desorption peak was observed at $230 \mathrm{~K}$, corresponding to molecules desorbing from the (111) terraces. Another desorption peak was observed at $345 \mathrm{~K}$ and is due to molecules desorbing from straight step edges. A third desorption peak at $385 \mathrm{~K}$ arises from desorption of R-3MCHO from kink sites. The assignment of these three features to desorption from terrace, step and kink sites has been made by comparison with the TPD spectra from $\mathrm{Cu}(111), \mathrm{Cu}(221)$ and $\mathrm{Cu}(533)$ surfaces that have only (111) terrace structures or (111) terraces separated by straight step edges.

The chiral sites on the $\mathrm{Cu}(643)$ surfaces are the kinks and are the sites that would be expected to exhibit enantiospecificity. Careful measurement of the peak desorption temperature $\left(\mathrm{T}_{\mathrm{p}}\right)$ for desorption of R-3-MCHO from the kink sites reveals enantiospecific difference of $\Delta \mathrm{T}_{\mathrm{p}}=3.5 \pm 0.8 \mathrm{~K}$ on the $\mathrm{Cu}(643)^{\mathrm{R}}$ and $\mathrm{Cu}(643)^{\mathrm{S}}$ surfaces. These give an estimate of the enantiospecific differences in the desorption energies of R-3- MCHO on the $\mathrm{Cu}(643)^{\mathrm{R}}$ and $\mathrm{Cu}(643)^{\mathrm{S}}$ surfaces of $\Delta \Delta \mathrm{E}_{\text {des }} \approx 0.25 \mathrm{kcal} / \mathrm{mole}^{23}$. This difference in desorption energy differences corresponds to a difference of roughly $30 \%$ in desorption rates at room temperature. Reflection-absorption infrared spectroscopy has been used to examine the orientation of R-3-MCHO adsorbed at the kinks on the $\mathrm{Cu}(643)^{\mathrm{R}}$ and $\mathrm{Cu}(643)^{\mathrm{S}}$ surfaces. Differences in the absorption intensities of the molecule at the two types of kinks indicate that it orientation is dictated by the chirality of the kinks ${ }^{24}$. There is now a growing body of evidence that the naturally chiral surfaces of metals have enantiospecific properties that could in principle be used as the basis for enantioselective chemical processes.

\section{Chiral imprinting of achiral surfaces}

While single crystal metals can expose chiral surfaces by cleavage along high Miller index crystallographic planes, it is also possible to induce chiral reconstructions on achiral single crystal surfaces by the adsorption of chiral molecules. This process is 
distinct from chiral templating. In the case of chiral templating the chirality of the surface is imparted by the inherent chirality of the template and would be removed if the template were to be removed from the surface. In the process of chiral imprinting naturally chiral structures are created in the metal surface that would persist even if the imprinting agent were to be removed.

Adsorbate induced reconstructions are observed on many single crystal surfaces, even in the presence of simple adsorbates such as hydrogen, carbon, oxygen, or nitrogen atoms $^{25}$. Larger molecules can also induce reconstructions of metal surfaces. For example, adsorption of the p-aminobenzoic acid onto the $\mathrm{Cu}(100)$ surface induces the formation of $(11,13,1)$ facets ${ }^{26}$. A more interesting reconstruction occurs when the chiral amino acid L-lysine is deposited onto the achiral $\mathrm{Cu}(100)$ surface ${ }^{27}$. L-lysine adsorption induces the formation of homochiral $(3,1,17)^{\mathrm{R}}$ facets with naturally chiral structures that are similar to those of the kinked $\mathrm{Cu}(643)^{\mathrm{R}}$ and $\mathrm{Cu}(643)^{\mathrm{S}}$ surfaces. The important point is that only $(3,1,17)^{\mathrm{R}}$ facets were formed on the $\mathrm{Cu}(100)$ surface. No $(3$ $117)^{\mathrm{S}}$ facets observed, indicating that the adsorption of L-lysine transforms the achiral $\mathrm{Cu}(100)$ surface into a naturally chiral surface. Another example has shown that 2,5,8,11,14,17-hexa-(tertbutyl)decacyclene (HtBDC) adsorption on the achiral $\mathrm{Cu}(110)$ surface leads to the formation of chiral pits with edges formed of kinked steps ${ }^{28}$. Careful examination of these chiral pits reveals that their edges expose chiral kink sites of the type observed on the $\mathrm{Cu}(643)^{\mathrm{R}}$ and $\mathrm{Cu}(643)^{\mathrm{S}}$ surfaces. Furthermore, the chirality of the kinks is dictated by the chirality of the adsorbed HtBDC. The important point in this example is that the chiral pits imprinted onto the $\mathrm{Cu}(110)$ surface by adsorption of HtBDC persist after the removal of the HtBDC. Thus imprinting appears to be a viable route to the production of naturally chiral metal surfaces.

\section{Kink formation on Stepped Cu surfaces}

Our work on the high Miller index surfaces of $\mathrm{Cu}$ has reveled that R-3-MCHO can induce the formation of kinks into step edges. The ideal structures of the $\mathrm{Cu}(533)$ and $\mathrm{Cu}(221)$ surfaces are shown in Figure 3. Both surfaces have (111) oriented terraces that are approximately 3 atoms wide separated by monatomic steps in the surface. The $\mathrm{Cu}(533)$ surface has straight (100) steps while the $\mathrm{Cu}(221)$ surface has straight (110) 
steps. Both surface have been prepared and cleaned in vacuum by cycles of inert argon ion sputtering followed by annealing to $1000 \mathrm{~K}$. Cleanliness of the surfaces was established by Auger electron spectroscopy and low energy electron diffraction was used to verify the orientation of the $\mathrm{Cu}(221)$ and $\mathrm{Cu}(533)$ surfaces. It should be noted that there is a mirror plane running perpendicular to the steps and thus these surface are achiral.

Desorption of R-3-MCHO from the $\mathrm{Cu}(533)$ and $\mathrm{Cu}(221)$ has been studied using TPD measurements two different procedures for adsorption. In one case the R-3-MCHO was adsorbed onto the surface with the surface at a temperature of $90 \mathrm{~K}$. The Cu sample was then heated as a constant rate of $1 \mathrm{~K} / \mathrm{sec}$ while using a mass spectrometer to measure the desorption rate of R-3-MCHO from the surface. The resulting TPD spectrum is a plot of $\mathrm{R}-3-\mathrm{MCHO}$ desorption rate as a function of temperature. The second procedure differed only in that the R-3-MCHO was adsorbed on the surface with the surface temperature held at $330 \mathrm{~K}$. The adsorption temperature has a profound effect on the nature of the desorption spectra that on obtains during subsequent heating of the surface.

Figure 4 shows TPD spectra obtained for desorption of 0.1 monolayer of R-3-MCHO from the $\mathrm{Cu}(533)$ surface following adsorption at both $90 \mathrm{~K}$ and at $330 \mathrm{~K}$. At a coverage of 0.1 monolayer only adsorption sites at the step edges are populated. The peak desorption temperature of $\mathrm{R}-3-\mathrm{MCHO}$ from the $\mathrm{Cu}(533)$ after adsorption at $90 \mathrm{~K}$ is 350 $\mathrm{K}$. This is consistent with our assignment of the peak at $345 \mathrm{~K}$ from the $\mathrm{Cu}(643)$ surface to desorption from the straight steps edges (Figure 2). After adsorption of R-3-MCHO onto the $\mathrm{Cu}(533)$ surface at $330 \mathrm{~K}$, however, desorption occurs at a temperature of $372 \mathrm{~K}$. This temperature is in the range of temperatures assigned to desorption of R-3-MCHO from kink sites on the $\mathrm{Cu}(643)$ surface. One possible explanation is that the elevated adsorption temperature allows the surface to reconstruct and spontaneously form kinks at the previously straight step.

The effect of adsorption temperature of $\mathrm{R}-3-\mathrm{MCHO}$ on the $\mathrm{Cu}(221)$ surface is similar to that observed on the $\mathrm{Cu}(533)$ surface. TPD spectra for R-3-MCHO desorption following adsorption on the $\mathrm{Cu}(221)$ surface at both $90 \mathrm{~K}$ and $330 \mathrm{~K}$ are shown in Figure 5. The desorption peak following adsorption of 0.1 monolayers of R-3-MCHO at $90 \mathrm{~K}$ is a bit more complex than on the $\mathrm{Cu}(533)$ surface. It exhibits a peak at $355 \mathrm{~K}$ with a 
shoulder at $375 \mathrm{~K}$. Exposure of the $\mathrm{Cu}(221)$ surface to R-3-MCHO at $330 \mathrm{~K}$ followed by desorption results in a TPD spectrum in which the dominant feature is a desorption peak at $375 \mathrm{~K}$. The spectrum obtained following adsorption at $90 \mathrm{~K}$ indicates that the steps have some residual kinks which have been present on the clean surface or may be present on as a results of R-3-MCHO adsorption. It is clear though that adsorption at $330 \mathrm{~K}$ results in a surface structure in which the steps are highly kinked.

The evidence presented suggests that adsorption of R-3-MCHO on the achiral $\mathrm{Cu}(221)$ and $\mathrm{Cu}(533)$ surfaces can result in the formation of kinks in the naturally straight step edges. Kinks in step edges are naturally chiral and could impart a net chirality to the surface if the kinks are formed such that they are predominantly of one handedness. Although the handedness of the kinks could be dictated by the chirality of the R-3MCHO there is no evidence in these data that this is the case. Furthermore, it does not appear that the kinks formed by R-3-MCHO adsorption are stable once the R-3-MCHO has been desorbed from the surface. Subsequent adsorption of R-3-MCHO at $90 \mathrm{~K}$ and desorption yield spectra similar to those shown in Figures 4 and 5 for adsorption at $90 \mathrm{~K}$. Nonetheless, these experiments have provided additional evidence of imprinting of chiral structures onto otherwise achiral surfaces and suggest that this is a potential route to the formation of chiral surfaces.

\section{Summary}

Chiral surfaces are used in a number of enantioselective chemical processes and will have growing importance in the production of enantiomerically pure materials such as

pharmaceuticals. Naturally chiral surfaces can be created from materials with naturally chiral bulk crystal structures or from achiral materials such as metals that are cleaved to expose planes that are naturally chiral. Alternatively, chiral surface can be made from achiral materials by either templating with chiral organic ligands or by imprinting. Imprinting can create structures on surfaces similar to those exposed by naturally chiral planes. 


\section{References}

(1) Hazen, R. M.; Sholl, D. S. Nature Materials, in press.

(2) Bonner, W. A.; Kavasmaneck, P. R.; Martin, F. S. Science 1974, 186, 4159.

(3) Hazen, R. M.; Filley, T. R.; Goodfriend, G. A. Proceedings of the National Academy of Sciences of the United States of America 2001, 98, 5487-5490.

(4) SOAI, K.; SATO, I.; SHIBATA, T. The Chemical Record 2001, 1.

(5) Lorenzo, M. O.; Haq, S.; Bertrams, T.; Murray, P.; Raval, R.; Baddeley, C. J. J. Phys. Chem. 1999, 103, 10661.

(6) Humblot, V.; Haq, S.; Muryn, C.; Hofer, W. A.; Raval, R. J. Am. Chem. Soc. 2002, 124.

(7) Jones, T. E.; Baddeley, C. J. Surface Science 2002, 2002, 453-467.

(8) Xu, Q.; Wang, D.; Wan, L.; Bai, C.; Wang, Y. J. Am. Chem. Soc. Comm. 2002, 124, 14300-14301.

(9) Carley, A. F.; Rajumon, M. K.; Roberts, M. W.; Wells, P. B. Journal of the Chemical Society, Faraday Transactions 2002, 91, 2167-2172.

(10) Fasel, R.; Cossy, A.; Ernst, K.-H.; Baumberger, F.; Greber, T.; Osterwalder, J. J. Chem. Phys. 2001, 115, 1020-1027.

(11) Williams, J.; Haq, S.; Raval, R. Surface Science 1996, 368, 303-309.

(12) Chen, Q.; Lee, C. W.; Frankel, D. J.; Richardson, N. V. PhysChemComm 1999, 9.

(13) Marti, E. M.; Barlow, S. M.; Haq, S.; Raval, R. Surface Science 2002, 501, 191202.

(14) Stacchiola, D.; Burkholder, L.; Tysoe, W. T. J. Am. Chem. Soc. 2002, 124, 89848989.

(15) Sholl, D. S.; Asthagiri, A.; Power, T. D. Journal of Physical Chemistry B 2001, 105, 4771-4782.

(16) Ahmadi, A.; Attard, G. Langmuir 1999, 15, 2420-2424.

(17) Power, T. D.; Asthagiri, A.; Sholl, D. S. Langmuir 2002, 18, 3737-3748.

(18) Power, T. D.; Sholl, D. S. Topics in Catalysis 2002, 18, 201-208.

(19) Perry, S. S.; Zhao, X. unpublished results.

(20) Gellman, A. J.; Horvath, J. D.; Buelow, M. T. J. Mol. Cat. A 2001, 167, 3-11.

(21) Horvath, J. D.; Gellman, A. J. J. Am. Chem. Soc. 2001, 123, 7953-7954.

(22) Horvath, J. D.; Kamakoti, P.; Sholl, D. S.; Gellman, A. J. Nature, submitted.

(23) Horvath, J. D.; Gellman, A. J. J. Am. Chem. Soc. 2002, 124, 2384-2392.

(24) Horvath, J. D.; Gellman, A. J. unpublished results.

(25) Titmuss, S.; Wander, A.; King, D. Chemical Reviews 1996, 96, 1291.

(26) Chen, Q.; Frankel, D. J.; Richardson, N. V. Langmuir 2001, 17, 8276-8280.

(27) Zhao, X. J. Am. Chem. Soc. 2000, 122, 12584-12585.

(28) Schunack, M.; Laegsgaard, E.; Stensgaard, I.; Johannsen, I.; Besenbacher, F. Angewandte Chemie, International Edition 2001, 40, 2623-2626. 


\section{Figure Captions}

Figure 1. Ball models of the ideal $\mathrm{Cu}(643)^{\mathrm{S}}$ and $\mathrm{Cu}(643)^{\mathrm{R}}$ surfaces. The structures are nonsuperimposable mirror images of one another and, therefore, are chiral. The structure is based on a (111) terrace separated by kinked steps. The step edges have a (100) structure while the single atom kinks have a (110) structure. Chirality arises from the sense of rotation from (111) terrace to (100) step edge to (110) kink as one looks down on the surfaces.

Figure 2. Temperature Programmed Desorption (TPD) of 1 monolayer of R-3-MCHO from the $\mathrm{Cu}(643)^{\mathrm{R}}$ surface. The inset is a ball model of the roughened $\mathrm{Cu}(643)^{\mathrm{S}}$ surface with R-3-MCHO molecules adsorbed at the terrace, the step edge and the kink sites. The step edge is highlighted for visual purposes only. Each peak in the TPD spectrum is assigned to desorption of R-3-MCHO from different sites on the roughened $\mathrm{Cu}(643)^{\mathrm{S}}$ surface, as indicated by the arrows.

Figure 3. Ball models of the $\mathrm{Cu}(221)$ and $\mathrm{Cu}(533)$ surfaces. Both surfaces have (111) terraces approximately 3 atoms wide separated by straight step edges. The (533) surface has straight (100) steps while the (221) surface has straight (110) steps. There is a mirror plane running perpendicular to the steps and thus thee surfaces are achiral.

Figure 4. TPD of 0.1 monolayers of $\mathrm{R}-3-\mathrm{MCHO}$ from $\mathrm{Cu}(533)$ following adsorption at $90 \mathrm{~K}$ and $330 \mathrm{~K}$. Adsorption of R-3-MCHO at $330 \mathrm{~K}$ results in a higher peak desorption temperature than was observed after adsorption at $90 \mathrm{~K}$. These results suggest that adsorbate induced reconstruction occurs during exposure of the surface to R-3-MCHO at $330 \mathrm{~K}$. The reconstruction causes the formation of kinks in the otherwise straight step edge. TPD spectra were collected while heating the surface at $1 \mathrm{~K} / \mathrm{sec}$ and monitoring the signal at $m / q=39$.

Figure 5. TPD of 0.1 monolayers of R-3-MCHO from the $\mathrm{Cu}(221)$ surface following adsorption at $90 \mathrm{~K}$ and $330 \mathrm{~K}$. Adsorption of R-3-MCHO at $330 \mathrm{~K}$ results in a higher 
peak desorption temperature than was observed after adsorption at $90 \mathrm{~K}$. These results suggest that adsorbate induced reconstruction occurs during exposure of the surface to R3-MCHO at $330 \mathrm{~K}$. The reconstruction causes the formation of kinks in the otherwise straight step edge. TPD spectra were collected while heating the surface at $1 \mathrm{~K} / \mathrm{sec}$ and monitoring the signal at $m / q=39$. 

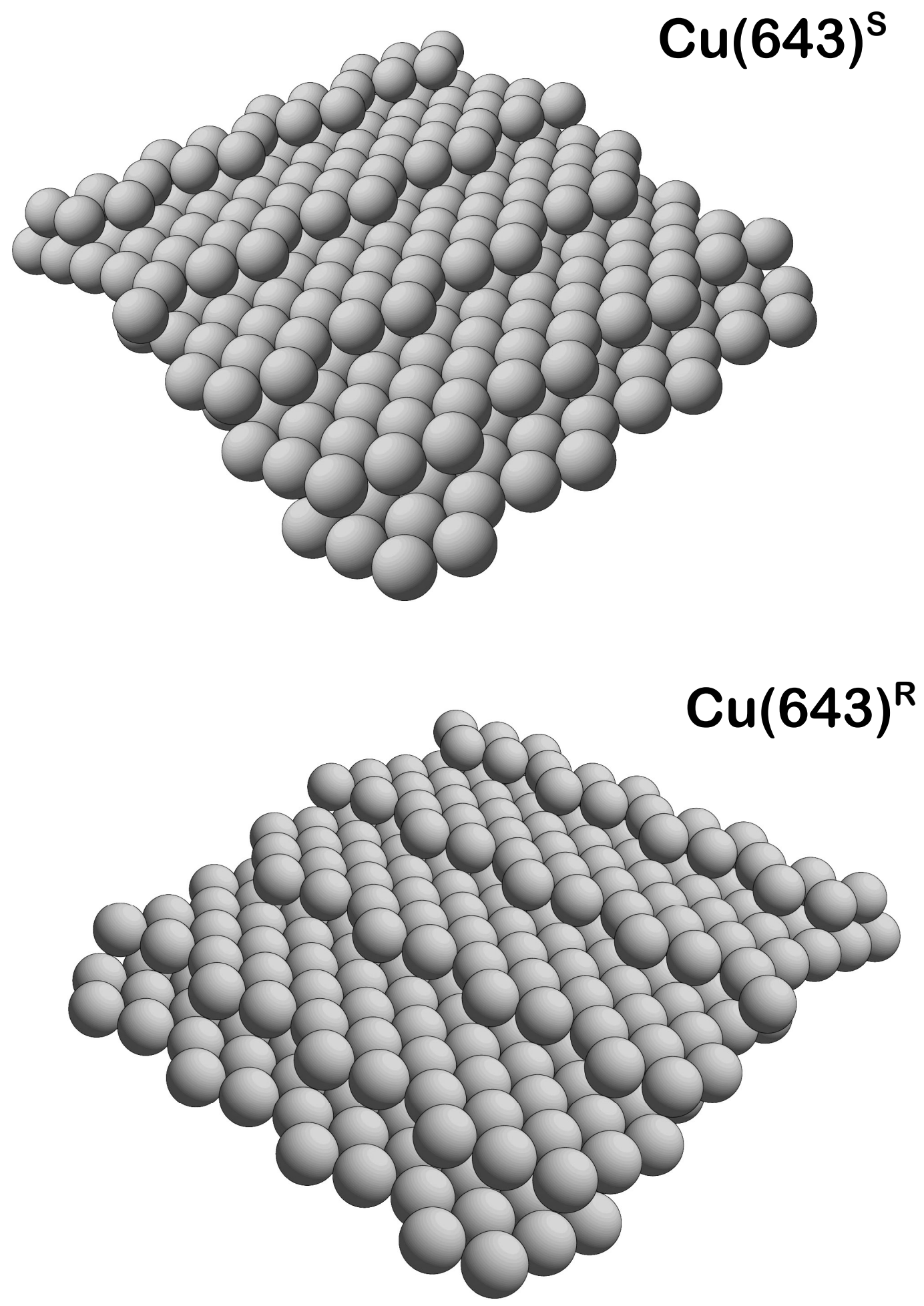

Newfigures/papers/

Chiral Surfaces T. in Cat./fig 


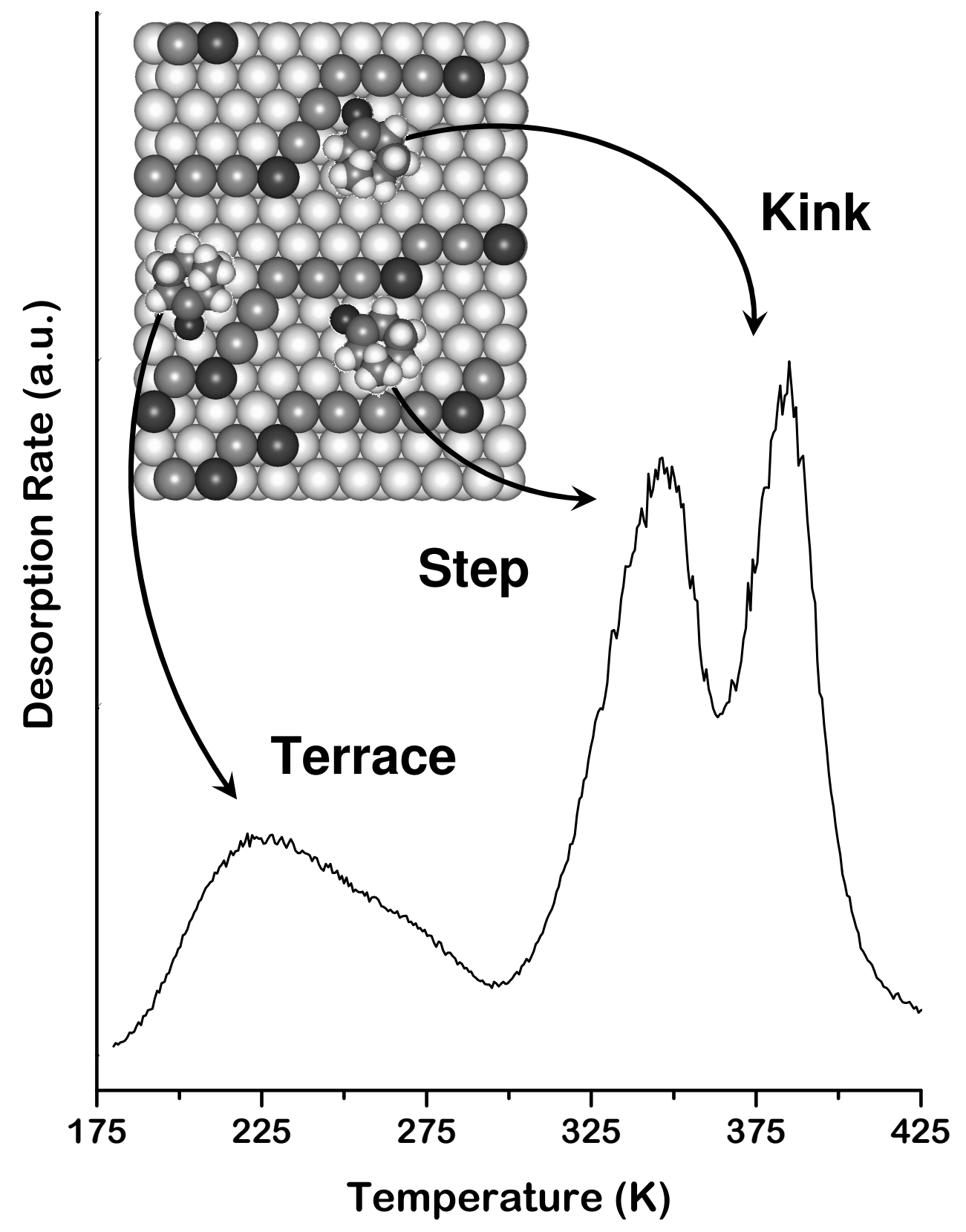

newfigures/papers/

Chiral Surfaces Top. Cat./fig 

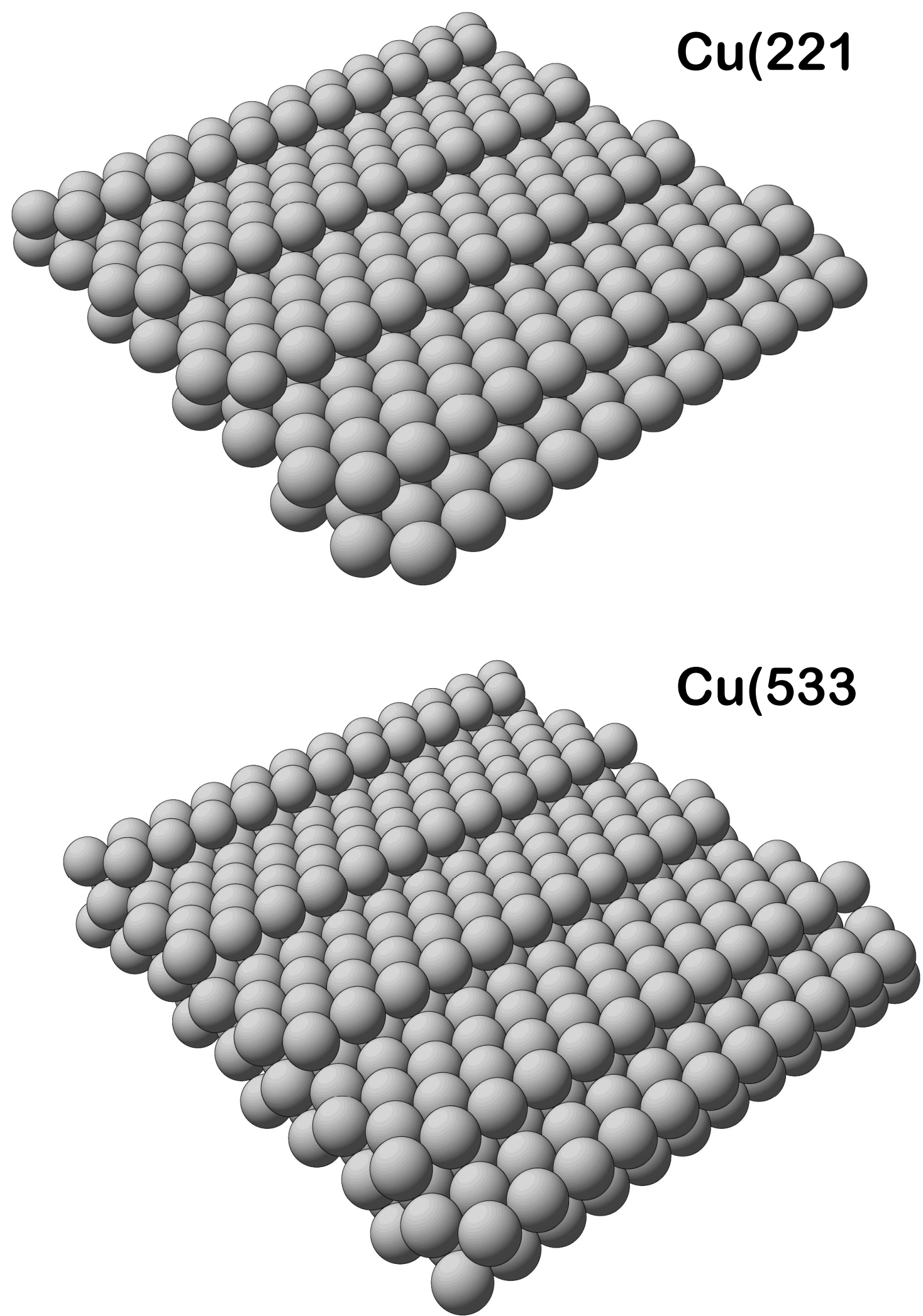

Newfigures/papers/

Chiral Surfaces Top./ Cat./fig 


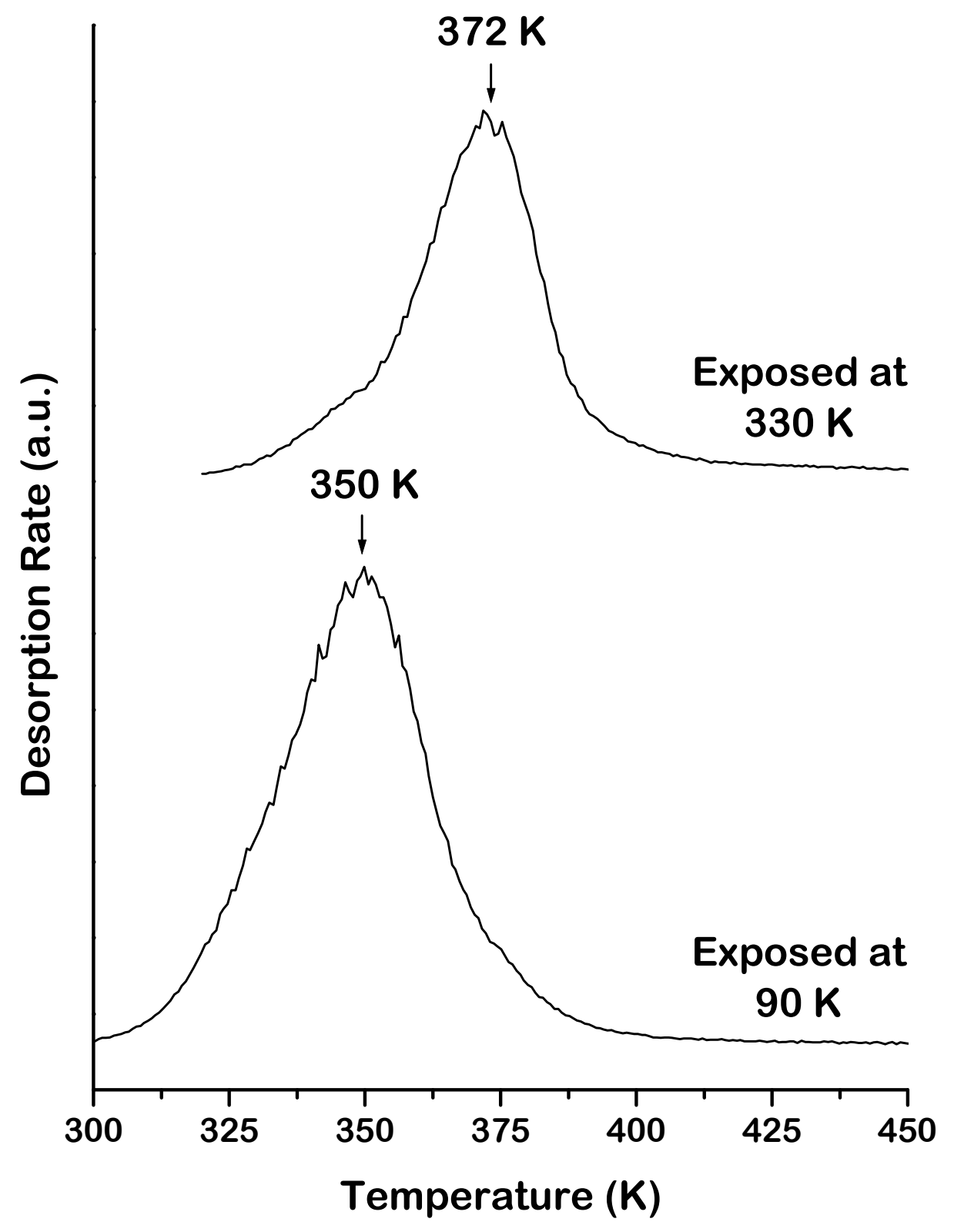

Chiral Surfaces Top. Cat./fig 4 


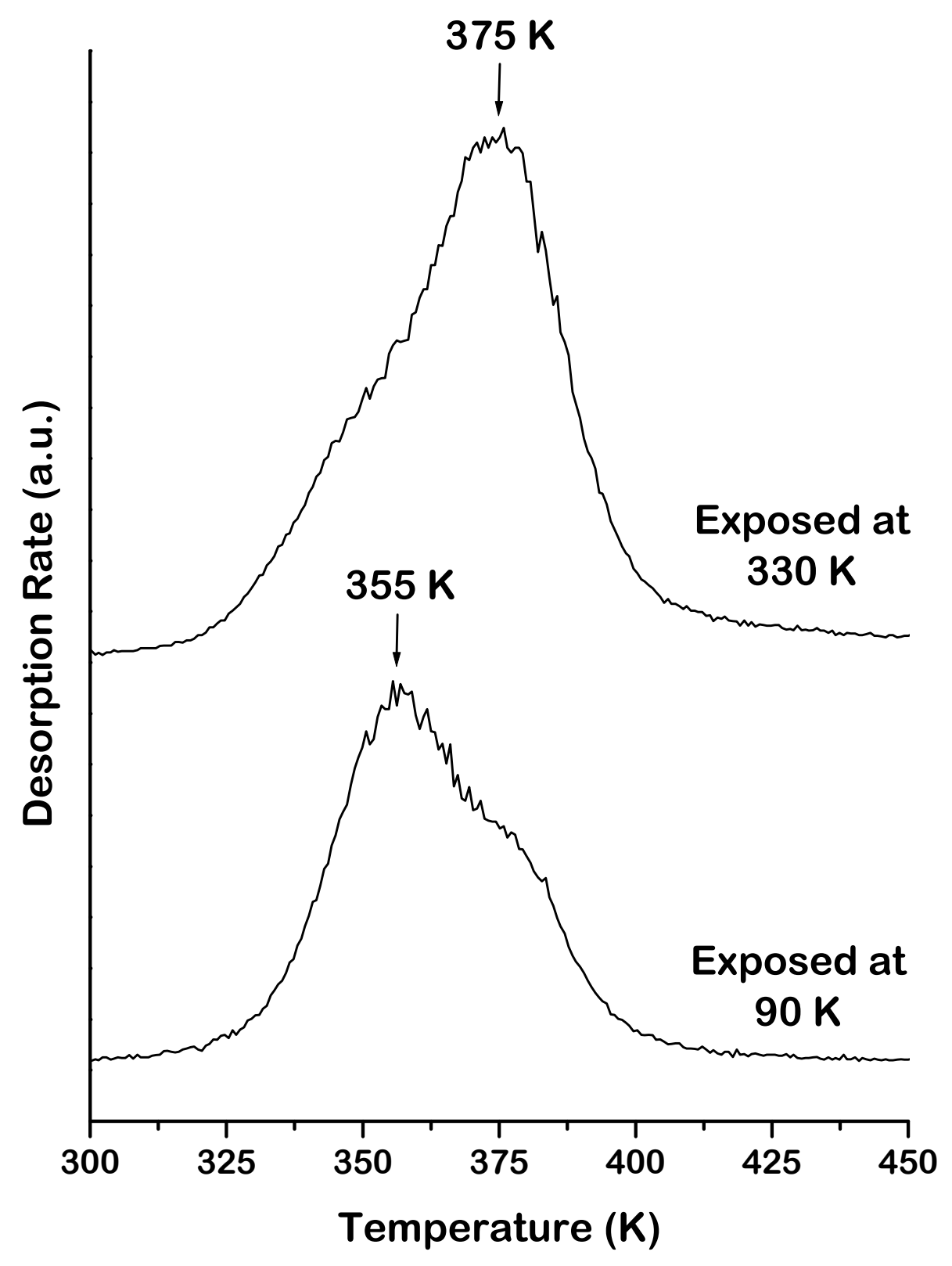

